\title{
PLAN DE CUIDADOS A UN PACIENTE CON BRONCOESPASMO SEVERO EN UNA REAGUDIZACIÓN DE EPOC
}

\section{CARE PLAN IN PATIENT WITH SEVERE BROCHOSPASM EXACERBATION OF EPOC}

Francisca Bernal Pérez.

\author{
Enfermera Hospital de Bellvitge. Hospitalet de Llobregat. \\ E-mail: paquibernal@gmx.es
}

\section{RESUMEN}

Presentamos caso de varón de 79 años con múltiples ingresos por exarcebación de EPOC y brocoespasmo severo con el objetivo de elaborar un plan de cuidados estandarizado para este paciente basándonos en la taxonomía enfermera NANDA, NIC, NOC. (1,2,3,4) $^{2}$

Con la presentación de este caso, pretendo desarrollar el lenguaje enfermero (5), para facilitar el trabajo de enfermería, así como garantizar y mejorar la continuidad de los cuidados desde su llegada a urgencias hasta su ubicación en planta.

Palabras clave: EPOC, broncoespasmo, plan de cuidados, lenguaje enfermero. 


\section{ABSTRACT}

\section{Care plan in patient with severe brochospasm exacerbation of EPOC.}

We present the case of a man of 79 years with multiple revenue brocoespasmo exarcebación severe EPOC and in order to develop a standard of care for this patient based on nursing taxonomy NANDA, NIC, NOC.

With the presentation of this case, I intend to develop a nursing language, to facilitate nursing work and to ensure and improve continuity of care from their arrival in the ED to its location on the ground.

Keywords: EPOC, bronchospasm, plan of care, nursing language.

\section{INTRODUCCIÓN}

La enfermedad pulmonar obstructiva crónica (EPOC) es en la actualidad un problema de salud pública muy importante, debido a su prevalencia y morbi-mortalidad. Muy ligada al tabaquismo, su prevalencia futura dependerá según el consumo de tabaco. Según la OMS, es la cuarta causa de muerte desde el año 2000, en el año 2020 se prevé que sea la tercera. ${ }^{(6)}$

La EPOC es un trastorno crónico caracterizado por la limitación al flujo aéreo a través de las vías respiratorias. Se produce una reacción inflamatoria pulmonar anómala muy frecuentemente asociada al humo del tabaco, partículas o gases tóxicos.

Dentro del término EPOC se incluyen dos formas clínicas:

1. Bronquitis crónica.

2. Enfisema pulmonar.

La EPOC en su etapa inicial suele ser asintomática y se manifiesta 20 o 30 años después del inicio del consumo habitual de tabaco. ${ }^{(7)}$ 
El paciente suele necesitar asistencia sanitaria muy frecuente, por lo que se necesitan muchos recursos asistenciales en el trato de estos pacientes. Por ser una enfermedad progresivamente invalidante, se deben tener en cuenta las repercusiones negativas sobre la calidad de vida del enfermo. Provocará sedentarismo y mayor tendencia a la depresión.

\section{CASO CLÍnICO}

Paciente de 79 años que acude a urgencias por EPOC con exarcebación e insuficiencia respiratoria hipercápnica.

\section{Antecedentes:}

- No alergias conocidas.

- Fumador activo 2 cigarrillos al día, previamente más.

- HTA en tratamiento médico.

- Dislipemia en tratamiento médico.

- Diabetes Mellitus tipo 2 en tratamiento con antidiabéticos orales (ADOS) .

- Probable nefropatía diabética con función renal estable aunque persiste proteinuria

- Arteriopatía diabética.

- Historia neumológica:

- EPOC grado 4 con oxigenoterapia domiciliaria de2lt 17-24 horas diarias. ${ }^{(8)}$

- Disnea habitual a pequeños esfuerzos.

- Múltiples ingresos por descompensaciones de EPOC reagudizada y insuficiencia cardiaca congestiva. ${ }^{(9)}$

- Coledocolitiasis en 2008 con extracción de cálculos por CPRE.

- Carcinoma escamoso T1N0 de cuerda vocal derecha en 1992. Actualmente libre de enfermedad. ${ }^{(10)}$

- BYPASS fémoro- poplíteo de miembro inferior izquierdo en 2002. 
- Situación basal: vive solo con cuidadora. Prácticamente dependiente para las actividades de la vida diaria (ABVD).

\section{Enfermedad actual:}

Paciente que acude a urgencias por presentar desde esta noche aumento de su disnea habitual, llegando a ser de mínimos esfuerzos y sibilancias audibles. Ortopnea. No tos ni expectoración. No edemas maleolares. La cuidadora afirma que ayer fumó seis cigarrillos.

\section{Exploración física:}

TA $150 / 85 \mathrm{mmHg}$

FC $80 \mathrm{lpm}$

FR $34 \mathrm{rpm}$

Ta $36 ; 0^{\circ} \mathrm{C}$

SAT02 90\% con $\mathrm{FiO} 2$ del $26 \%$.

Peso: $74 \mathrm{~kg}$

Altura: $1,65 \mathrm{~cm}$.

Hemodinámicamente estable, consciente y orientado en tiempo, espacio y persona. Hipofonesis generalizada, sibilancias diseminadas.

\section{Exploración complementaria:}

- Gasometría arterial destacando acidosis respiratoria.

- RX tórax: cardiomegalia. Signos de edema intersticial.

- ECG: Bloqueo de rama derecha.

\section{Evolución clínica:}

Paciente que con los antecedentes descritos acude por descompensación de EPOC con insuficiencia respiratoria hipercápnica que empeora tras tratamiento inicial en urgencias y precisa ventilación mecánica no invasiva. Tras valoración con pneumólogo de guardia se orientan como diagnósticos:

1. Brocoespasmo severo.

2. Reagudización de EPOC. 
3. Insuficiencia respiratoria hipercápnica.

4. Acidosis respiratoria.

Se decide su ingreso en la planta de neumología para estabilización y evolución con tratamiento broncodilatador.

\section{VALORACIÓN DE ENFERMERÍA POR NECESIDADES BÁSICAS.}

1.Necesidad de respirar: Fumador activo de entre 2 y 6 cigarrillos diarios, previamente más. Disnea a pequeños esfuerzos, oxigenoterapia domiciliaría, ortopnea precisa dos almohadones para dormir. Tratamiento con broncodilatadores que no realiza correctamente. A su ingreso vías respiratorias con sibilancias, taquipnea y saturaciones de oxígeno bajas. Rx de tórax que evidencia cardiomegalia y signos de edema intersticial. Gasometría arterial que determina acidosis respiratoria e insuficiencia hipercápnica.

2.Necesidad de nutrición e hidratación: A su ingreso pesa $74 \mathrm{~kg}$ y mide $1,65 \mathrm{~cm}$. IMC de 27,18 que indica sobrepeso. Edemas en extremidades inferiores y cianosis en dedos de las manos. En casa no sigue una dieta equilibrada, excesos de hidratos de carbono y grasas. Manifiesta que si siguiera las recomendaciones alimenticias de los médicos, no podría comer de nada. Dificultad en la masticación por pérdida de piezas dentales. No le gusta la comida del hospital.

3.Necesidad de eliminación: Ritmo intestinal normal. Aspecto y consistencia normales. En estudio por probable nefropatía diabética con función renal estable. Ligera pérdida de orina cando tiene ataques de tos.

4.Necesidad de movimiento: Dependiente para las ABVD. Disnea habitual a pequeños esfuerzos, por lo que casi todo el día está sentado en un sillón.

5.Necesidad de descanso y sueño: Descanso no reparador, se despierta 
multitud de veces en la noche, precisa de almohadones para descansar, ortopnea. Se levanta cansado. Manifiesta tener miedo de no despertar más, cuando tiene exacerbaciones.

6.Necesidad de vestirse y desvestirse: Dependiente, le ayuda su cuidadora habitual. Se cansa demasiado. Utiliza ropa cómoda.

7.Necesidad de termorregulación: Manifiesta tener siempre frío. Afebril durante su estancia hospitalaria.

8.Necesidad de higiene y protección de la piel: Presenta aspecto cuidado, hidratado. Ducha 3 veces por semana e higiene bucal incorrecta. Precisa ayuda de la cuidadora. Portador de vía periférica corta. Durante su ingreso ha sido necesario varias veces el recambio por salida accidental del catéter. Difícil acceso venoso por venas tortuosas y frágiles.

9.Necesidad de evitar peligros: Consciente y orientado. Colaborador. Atiende a las explicaciones que se le dan. Está angustiado por su estado de salud y refiere que le queda poco tiempo de vida. Continua fumando porque es el único placer que tiene junto con la comida por eso no realiza las recomendaciones de sus médicos en este aspecto.

10.Necesidad de vivir según sus creencias y valores: Católico no practicante.

11. Necesidad de comunicarse: Conserva todos los sentidos. Demanda constante de atención. Se define como una persona introvertida, su único apoyo es la cuidadora, pero ella no es su familia.

12.Necesidad de trabajo y realización: Pensionista. Trabajó en la automoción como mecánico. Le gustaba estar rodeado de grasa. Añora aquellos tiempos en los que se manejaba sólo. Ahora se encuentra inválido. 
13.Necesidad de recrearse: Desde su enfermedad, apenas sale, sólo para ir al médico en contadas ocasiones. Se cansa demasiado y le da apuro salir con el oxígeno por la calle. Se ha hecho de su casa su santuario y en él se encuentra protegido. Apenas tiene familia que no lo vienen a visitar porque también son mayores. Se encuentra solo.

14.Necesidad de aprender: Estudios de mecánico, capacidad de comprensión normal. Conoce el diagnóstico, pero no muestra interés por los cambios que deberá hacer al alta. No realiza bien la técnica de los inhaladores.

\section{SÍNTESIS DE LOS DATOS}

Paciente de 79 años que vive solo con su cuidadora habitual durante el día, por la noche permanece solo. Está jubilado desde los 62 años por enfermedad y es pensionista.

No sigue una dieta adecuada, abusa de los hidratos de carbono y grasas y come dieta sódica a pesar de los antecedentes patológicos que padece: IAM, diabetes, HTA. Continua fumando, pese a llevar oxigenoterapia domiciliaria y continuas descompensaciones respiratorias. No realiza bien la técnica de los inhaladores. En todo momento se muestra preocupado por su evolución, pero manifiesta que no está dispuesto a realizar ciertos sacrificios: fumar y comer porque es lo único que le queda.

Refiere no descansar a gusto, se despierta muchas veces por la noche, se encuentra angustiado y deprimido.

Desde su enfermedad, apenas sale de su casa y necesita ayuda para todo, porque se agota muy rápido.Le falta el aire en cualquier actividad, por eso se ha acostumbrado a ser más dependiente de lo que es en realidad. Le da vergüenza salir con el oxígeno por la calle.

Muestra interés a simple vista de los consejos y explicaciones, pero manifiesta que ya hará en casa las recomendaciones necesarias. En el hospital sigue una dieta equilibrada, pero no le gusta la comida. Mantiene dificultad en la masticación por pérdida de piezas dentales. 
Tras valoración, recogemos los diagnósticos más importantes que se observan en este paciente, siguiendo la taxonomía enfermera NANDA, NIC, NOC.

\section{Disnea $\mathrm{r} / \mathrm{c}$ deterioro intercambio gaseoso y $\mathrm{m} / \mathrm{p}$ taquicardia $\mathrm{y}$ taquipnea.}

NOC: Resultados: Realizará respiraciones eficaces y no presentará signos y síntomas de mala perfusión.

0403 Estado respiratorio: ventilación.

0402 Estado respiratorio: Intercambio gaseoso.

0410 Estado respiratorio: Permeabilidad vías respiratorias.

Indicadores:

040313 Disnea de reposo.

040316 Dificultad respiratoria.

040211 Saturación de oxígeno.

040206 Cianosis.

\section{NIC: Intervenciones:}

3350 Monitorización respiratoria: mediante control de saturación de 02 que deberá ser superior o cercana al 90\% con la menor Fi02 posible. Control de estado mental del paciente, un sopor, una agitación son indicativos de hipoxemia.

2300 Administración de medicación: Broncodilatadores agonistas beta2, metilxantinas, anticolinérgicos y esteroides, preferiblemente en nebulización. Utilización de beta-2 adrenérgicos como el salbutamol y la terbutalina. Observar la eficacia de la aerosolterapia y la tolerancia de ésta por el paciente. Uso de corticoides por el efecto antiinflamatorio y regulador mucociliar. Generalmente se utiliza metilprednisolona o hidrocortisona para una acción más rápida. Se deberá controlar la glicemia del paciente, el uso de corticoides junto a una descompensación patológica en el organismo eleva las cifras de glicemia. 
3320 Oxigenoterapia: Con el fin de evitar la hipoxia, se deberá conseguir una p02entre 55-60mmh. Tras la administración de 02 existe el riesgo de aumentar la PCO2 al disminuir el estímulo que produce la hipoxemia sobre el centro respiratorio. Sin embargo el objetivo es corregir la hipoxemia aún a riesgo de aumentar la hipercapnia, ya que la primera representa mayor riesgo de sufrir parada cardiorrespiratoria.

03140 Manejo de las vías aéreas: Si el paciente presenta criterios clínico-gasométricos de gravedad está indicada la intubación endotraqueal y la ventilación mecánica.

Actividades:

- Vigilancia parámetros respiratorios, ritmo, profundidad .

- Anotar las características de la tos y enseñar a toser de manera efectiva.

- Colocar paciente en posición Fowler para permitir máxima ventilación.

- Administrar broncodilatadores si procede.

- Administrar aerosolterapia si procede.

- Enseñar uso de inhaladores si es el caso.

- Administrar oxigenoterapia según prescripción facultativa.

- Controlar eficacia de la oxigenoterapia mediante pulsioximetría y gasometría arterial.

- Prevención tromboembólica.

- Rehabilitación respiratoria que incluyan ejercicios de entrenamiento muscular, fisioterapia, educación sanitaria y tratamiento dietético.

00092 Intolerancia a la actividad $\mathrm{r} / \mathrm{c}$ entre desequilibrio y demandas de oxígeno y $\mathrm{m} / \mathrm{p}$ disnea, aumento de frecuencia cardiaca y respiratoria anormales en respuesta a la actividad.

DOC:Resultados: Realizará sus autocuidados dentro de sus posibilidades. 
Presentará un aumento progresivo de tolerancia a la actividad. 0005 Tolerancia a la actividad.

Indicadores:

000508 Esfuerzo respiratorio después de caminar. 00510 Distancia de caminata.

NIC: Intervenciones.

0180 Manejo de energía: Enseñar al paciente a marcar el ritmo de las actividades diarias en función de su ciclo respiratorio.

1803 Ayuda en los autocuidados: alimentación. Fomentar el descanso antes de comer. Uso de gafas nasales. Evitar beber una hora antes de las comidas. Considerar el uso de soportes nutricionales para aumentar la energía. Utilización de respiración con los labios fruncidos y respiración diafragmática.

1804 Ayuda en los autocuidados: aseo/eliminación.

1802 Ayuda en los autocuidados: vestir/ arreglo personal

1801 Ayuda en los autocuidados: baño/higiene. Mantendrá una higiene adecuada y se mostrará satisfecho con su aspecto personal.

Actividades:

- Indagar sobre las limitaciones físicas del paciente.

- Animar a la movilización activa y pasiva en la cama.

- Promover la sedestación.

- Vigilar parámetros cardiorrespiratorios a la actividad ( taquicardia, taquipnea, sudoración, disnea).

- Colocar los objetos de uso frecuente al alcance del paciente.

- Ayudar en la deambulación, si procede con dispositivos de ayuda (caminador, bastón).

- Animar a realizar ejercicios según tolerancia.

- Ayudarlo en los autocuidados de aseo, vestir y baño.

- Vigilar la integridad cutánea. 
- Implicar a la familia siempre que sea posible.

- Remitir al fisioterapeuta si procede.

\section{Alteración del patrón del sueño $r / c$ disnea, ortopnea y $\mathrm{m} / \mathrm{p}$ por múltiples despertares y descanso no reparador.}

> NOC: Resultados: El paciente dormirá un mínimo de 6 horas seguidas y verbalizará levantarse descansado.

0003 Descanso.

0004 Sueño.

Indicadores:

000301 Tiempo de descanso.

000303 Calidad del descanso.

000401 Horas de sueño.

000404 Calidad del sueño.

NIC: Intervenciones.

1850 Fomentar el sueño: Aconsejar medidas inductoras al descanso nocturno como infusiones, ambiente tranquilo, temperatura agradable. Evitar el uso de estimulantes cuatro horas antes de acostarse y evitar ingestas copiosas una hora antes de irse a dormir.

Actividades:

- Planificar los cuidados enfermeros en horas donde no se altere el ciclo regular sueño/vigilia.

- Animar al paciente a establecer una rutina a la hora de irse a la cama.

- Evitar la ingesta de alimentos y bebidas que puedan interferir en el sueño.

- Procurar ambiente tranquilo (luz, ruidos, temperatura, colchón, cama) para favorecer el sueño. 
00078 Manejo inefectivo del régimen terapéutico $\mathrm{r} / \mathrm{c}$ déficit de conocimientos, complejidad del régimen terapéutico y $\mathrm{m} / \mathrm{p}$ conductas no apropiadas o adaptativas y verbalizaciones del propio paciente.

NOC: Resultados: El paciente o la cuidadora verbalizarán los problemas que le produce el tratamiento, diseñando un plan de cuidados de acuerdo al tratamiento y a la modificación de hábitos.

1061 Conducta de cumplimiento. Verbalizarán signos y síntomas de alerta de agravamiento de la enfermedad.

1608 Control de síntomas.

1609 Conducta terapéutica: enfermedad o lesión.

1813 Conocimiento: régimen terapéutico. Conocerán los medicamentos a administrar, efectos secundarios y efectividad de los mismos.

Indicadores:

160101 Confianza en el profesional sanitario.

160103 Comunica seguir la pauta prescrita.

160106 Modifica la pauta prescrita por el profesional enfermero.

NIC: Intervenciones:

4360 Modificación de la conducta.: Evitar hábitos insanos como el tabaco.

4470 Ayuda en la modificación de sí mismo. Proporcionarle mecanismos adaptativos para la deshabituación del hábito tabáquico.

4350 Manejo de la conducta.

5510 Educación sanitaria.

Actividades:

- Establecer una relación terapéutica basada en la confianza y respecto, demostrando empatía. 
- Identificar la conducta que ha de modificarse.

- Ayudar a identificar sus puntos fuertes y reforzarlos.

- Evaluar nivel de conocimientos del paciente con el proceso de enfermedad.

- Comentar los cambios en el estilo de vida que serán necesarios afrontar para evitar posibles complicaciones

- Educarle en una nutrición correcta: dieta equilibrada en hidratos de carbono y dieta libre de sal y grasas.

- Reforzar dieta para disminuir sobrepeso.

- Instrucción sobre los signos de alarma y las actuaciones a seguir ante ellos.

- Enseñanza sobre los medicamentos prescritos, propósito y acción.

- Instruirle en cuanto a dosis, duración y forma correcta de administración.

- Adecuarle cámara inhalatoria para los inhaladores y explicar forma correcta de realización.

- Establecer ayuda con otros profesionales sanitarios si procede ( asistente social, trabajador social).

- Educar al cuidador ocasional en las conductas a seguir.

00099 Mantenimiento inefectivo de la salud $\mathrm{r} / \mathrm{c}$ patología respiratoria y $\mathrm{m} / \mathrm{p}$ consumo de tabaco habitual.

DOC: Resultados: El paciente dejará de fumar y encontrará mecanismos de adaptación alternativos.

1805 Conocimiento: conducta sanitaria.

Indicadores:

180506 Descripción efectos sobre la salud del consumo de tabaco.

NIC: Intervenciones:

4490 Ayuda para dejar de fumar. Proporcionarle información y consejos de cómo dejar de fumar. 
Actividades:

- Registrar el estado actual y el historial de la actividad de fumar.

- Aconsejar al fumador de forma clara y consistente dejar de fumar.

- Explicar los efectos del tabaco sobre las vías respiratorias.

- Explicarle los riesgos de fumar cerca de la toma de oxígeno.

- Informarle sobre los productos sustitutivos de la nicotina.

- Ayudarle a fijar una fecha para dejar de fumar.

- Referirle que los síntomas físicos de la abstinencia de la nicotina son temporales.

- Facilitar la autorresponsabilidad.

\section{Riesgo de infección r/c procedimientos invasivos (catéter venoso periférico)}

NOC: Resultados: El paciente conocerá y comunicará los signos y síntomas de infección relacionados con el cateterismo que puedan aparecer. El profesional de enfermería mantendrá unas medidas de seguridad para evitar las complicaciones del mismo .

1902 Control del riesgo.

Indicadores:

190216 Reconoce cambios en el estado de salud.

NIC: Intervenciones:

6540 Control de infecciones. Vigilancia de signos y síntomas de infección y del punto de inserción continua .

2240 Mantenimiento de dispositivos de acceso venoso periférico. Protocolo hospitalario sobre el mantenimiento y cuidados de vías periféricas.

Actividades:

- Instaurar precauciones universales. 
- Instruir al paciente a cerca de los signos de infección.

- Mantener técnica aséptica en la manipulación de catéteres.

- Cambiar los sistemas, dispositivos y tapones según protocolo.

- Curar con antisépticos el punto de inserción de los catéteres.

- Vigilar permeabilidad de los accesos venosos.

- Cambiar apósitos de protección según protocolo.

- Observar estado de la piel ( tumefacción, eritema, fiebre) que podrían ser indicadores de proceso infeccioso.

\section{CONCLUSIONES}

Cuando enfermería utiliza un plan de cuidados estandarizados, está utilizando una guía metodológica que facilita la continuidad de los cuidados y garantiza la calidad asistencial. Permiten desarrollar nuestra profesión de una manera eficaz porque se unifican criterios de atención, favorecen la comunicación con los pacientes y el resto de profesionales de la salud y fomentan la formación y la profesionalidad enfermera.

Son planes más ágiles, destacando la eficacia en la planificación y ejecución de los cuidados, así como la evaluación de nuestras intervenciones. La utilización de estas guías garantizan unos cuidados integrales y básicos para el cuidados de nuestros pacientes. ${ }^{(11)}$

\section{BIBLIOGRAFÍA:}

1. NANDA internacional. Diagnósticos enfermeros: definiciones y clasificación (2009-2011) Ed Elsevier: Madrid.

2. Clasificación de Resultados de Enfermería (NOC), Madrid España, 2006. Ed Elsevier.

3. Clasificación de Intervenciones (NIC), Madrid España, 2006. Ed Elsevier.

4. McCloskey J, Bulechek GM, Clasificación de Intervenciones de Enfermeria (NIC) , 4a Ed Elsevier;2005.

5. Carpenito LJ. Diagnósticos de Enfermería. Aplicaciones a la práctica clínica 9a ed. Madrid: McGraw-Hill Interamericana; 2002.

6. Calle Rubio M, Lobo Alvarez MA, Marzo Castillejo M, Mascarós Balaguer E. 
Atención integral al paciente con EPOC. Desde la atención primaria a la especializada. Barcelona: SEMFYC-SEPAR; 2010.

7. Lumbreras García G. Enfermedad pulmonar obstructiva crónica(EPOC). Madrid: Sanidad y Ediciones; 2008.

8. J.A Barberá, G. Peces-Barba, AG.N Agustí, JL. Izquierdo, E. Monsó,T. Montemayor y JL Viejo. Grupo de trabajo de la Sociedad Española de Neumología y Cirugía Torácica( SEPAR). Guía clínica para el diagnóstico y tratamiento de la Enfermedad pulmonar obstructica crónica. Arch Bronconeumol 2001; 37: 297-316.

9. Jurado Gómez B, Torres Murillo JM, Jiménez Murillo L, Berlanga Jiménez A, Degayón Tojo $\mathrm{H}$, et al. Epoc descompensada. En: Jiménez Murillo, L, Montero PérezFJ. Medicina de Urgencias y Emergencias. Guía diagnóstica y protocolos de actuación. Madrid: Elsevier España S.A,2004 p: 239-44.

10. Armstrong WB, Vokes D E, Maisel RH.Malignant tumors of the larynx In: Flint PW, Haughey $\mathrm{BH}$, Lund VJ, et al,eds. Cumming Otolaryngology Head and Neck Surgery . $5^{\text {th }}$ ed St Louis, Mo: Mosby Elsevier, 2010,chap 107.

11. Guía de Buenas Prácticas: Cuidados de Enfermería para la disnea: La sexta constante útil en personas con EPOC. Asociación de Enfermeras profesionales de Notario. Marzo 2005. 